\title{
Angiogenesis and Lymphangiogenesis: New Molecular Targets for the Treatment of Cancer and Inflammation
}

\author{
Michael Detmar*
}

\begin{abstract}
In order to grow beyond minimal size, tumors need to induce the growth of new blood vessels (angiogenesis), and systemic blockade of angiogenesis has been recently approved for the treatment of select types of human cancers. We identified the natural angiogenesis inhibitors thrombospondin-1 (TSP-1) and TSP-2 as potent inhibitors of ultraviolet light-induced skin damage and of cancer formation and metastasis, leading to new molecular approaches for chemoprevention. We also established the new concept of active tumor lymphangiogenesis, and we identified tumor lymphangiogenesis as a novel prognostic indicator for metastasis and patient survival of human cancers. We discovered VEGF as a major pathogenetic factor in chronic inflammation and established anti-angiogenic therapy as a novel strategy to treat inflammatory diseases. The overall goals of our ongoing research are to identify and to characterize molecular targets controlling inflammatory and tumor angiogenesis and lymphangiogenesis, using genetic disease models together with genomics approaches and chemical library screens, and to characterize genetic polymorphisms to determine disease susceptibility and to develop individualized therapeutic strategies.
\end{abstract}

Keywords: Angiogenesis · Cancer · Inflammation · Lymphangiogenesis · Pharmacogenomics

\section{Tumor Angiogenesis - A Novel Therapeutic Target}

Cancer research and therapy has predominantly focused on the genetic alterations and the abnormal growth of cancer cells. However, recent evidence indicates that non-cancerous cells in the tumor-surrounding stroma play an essential role in cancer progression. In particular, tumors need to induce the growth of new blood vessels (angiogenesis) in order to grow beyond minimal size and to metastasize. Vascular endothelial growth factor-A (VEGF-A) is the major tumor angiogenesis factor [1], promoting tumor growth, invasion, and organ metastasis (Fig. 1), and systemic anti-
${ }^{\star}$ Correspondence: Prof. Dr. M. Detmar Swiss Federal Institute of Technology Zürich Institute of Pharmaceutical Sciences

Department of Chemistry and Applied Biosciences Wolfgang Paulistrasse 10, $\mathrm{HCl} \mathrm{H} 303$

ETH Hönggerberg

$\mathrm{CH}-8093$ Zürich

Tel.: +41446337361

Fax: +41446337363

E-Mail: michael.detmar@pharma.ethz.ch

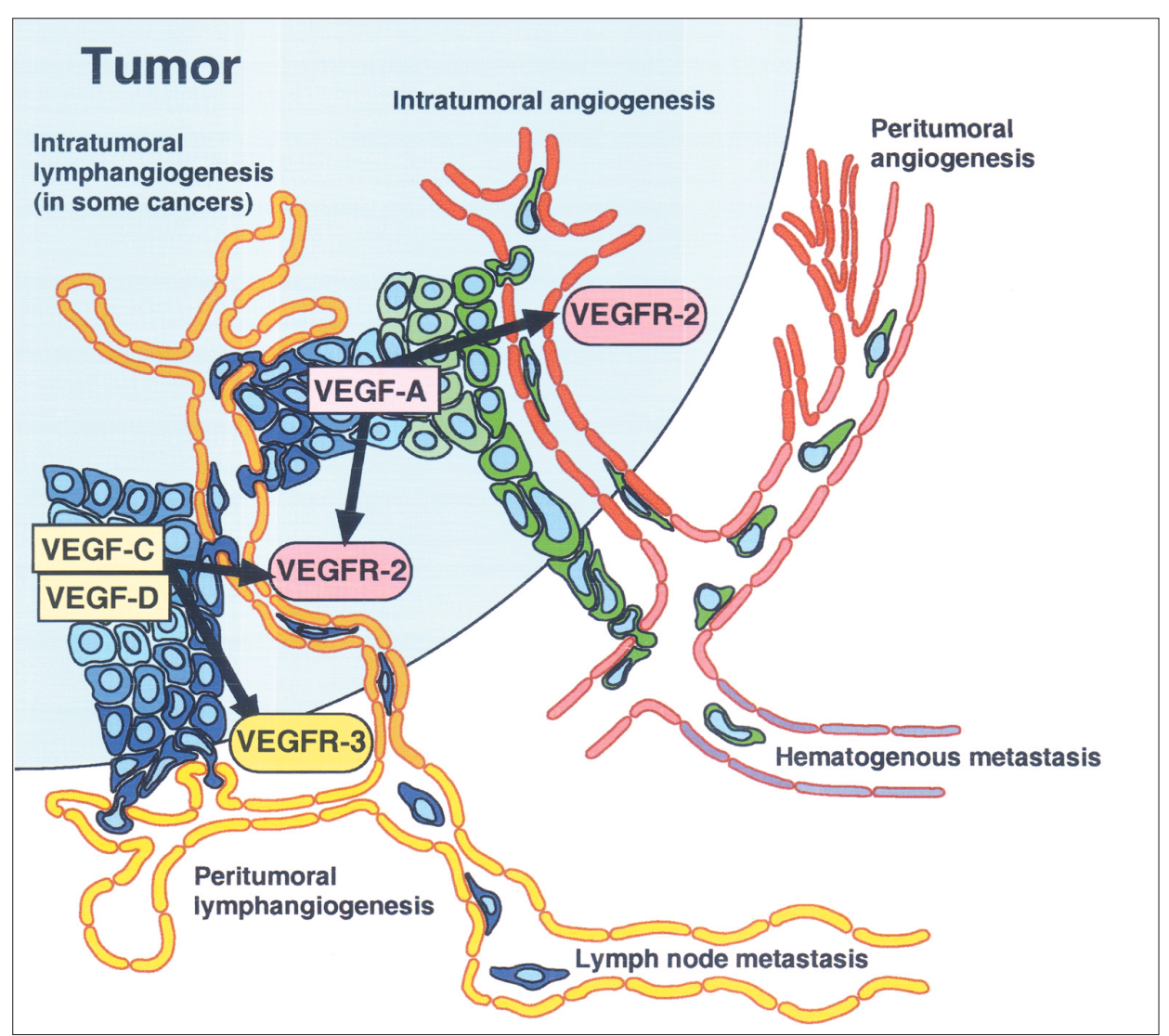

Fig. 1. Schematic representation of the molecular control of angiogenesis and lymphangiogenesis during cancer progression and metastasis 
angiogenic therapy with the VEGF-A blocking humanized antibody bevacizumab (Avastin ${ }^{\circledR}$ ) has been approved in 2004 for the therapy of human colon cancer patients. We have identified the matricellular molecule thrombospondin-2 (TSP-2; Fig. 2 ) as a novel, potent inhibitor of cancer growth and tumor angiogenesis [2]. We found that stromal upregulation of TSP-2 occurs during multi-step carcinogenesis as a natural anti-tumor host defense mechanism, and that carcinogenesis was enhanced in TSP-2 knockout mice but diminished in TSP-2 transgenic mice [3]. We are currently exploring mechanisms of TSP-2 action in vitro and in vivo, using transcriptional profiling and signal transduction studies, as well as functional studies in genetic mouse models. We have expressed recombinant human TSP-2 fragments and have confirmed their antitumoral activity on different human cancer types after systemic injection [4]. More recently, we have established the first proof of principle for the feasibility of cell-based systemic antiangiogenic gene therapy for treating cancer [5], using intraperitoneal implantation of biodegradable scaffolds that were populated with (ex vivo) TSP-2 transduced mouse fibroblasts originally obtained from skin biopsies. Further studies to potential- ly develop this therapeutic strategy for application in cancer patients (Fig. 3) are on the way.

\section{Chemoprevention of Ultraviolet-B- induced Skin Aging and Carcino- genesis}

In transcriptional profiling studies of tissues obtained during the distinct steps of skin carcinogenesis and after ultraviolet $B$ (UVB) irradiation, we have identified the natural angiogenesis inhibitor thrombospondin-1 (TSP-1) as one of the most potently downregulated genes. Based upon these findings, we developed the first transgenic mouse model for targeted overexpression of an angiogenesis inhibitor and demonstrated that TSP-1 inhibited multistep carcinogenesis and organ metastasis [6], and also completely prevented UVBinduced photodamage and aging of the skin [7]. We have established a high-throughput cell-based TSP-1 reporter gene assay and have used this screening assay to identify natural compounds which enhance TSP-1 gene expression in epidermal keratinocytes. This technology has been patented and has led to the commercial launch of a new antiaging product in 2003 . To identify defined

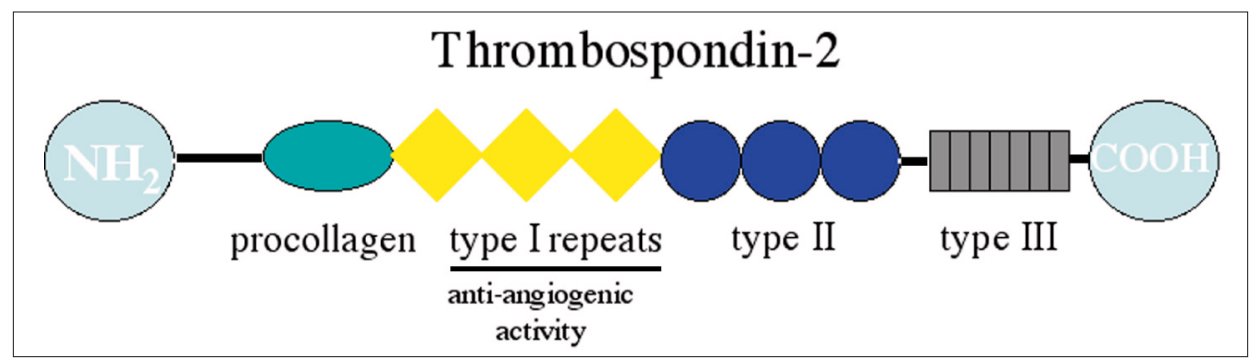

Fig. 2. Schematic representation of the modular thrombospondin-2 molecule, a potent natural inhibitor of tumor angiogenesis

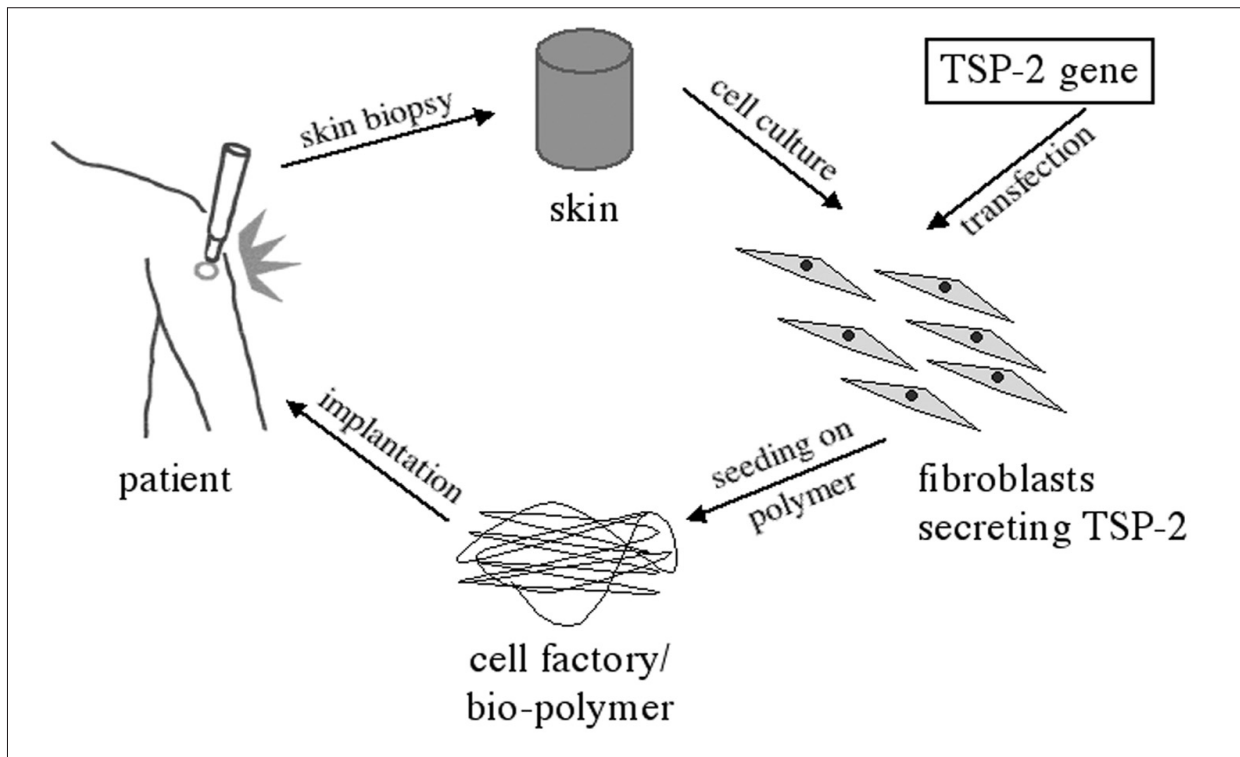

Fig. 3. Schematic representation of the principles of systemic, cell-based, anti-angiogenic gene therapy with thrombospondin-2

small synthetic TSP-1-inducing molecules that might be used clinically for the chemoprevention and/or treatment of human cancers, we are currently screening chemical libraries in high-throughput assays. We are also interested whether specific polymorphisms of the TSP-1 gene might be detected in patients with high cancer incidence.

\section{Tumor Lymphangiogenesis and Metastasis}

Metastasis to regional lymph nodes represents the first step of tumor dissemination in most human cancers and serves as a major prognostic indicator for disease progression. However, little is known about the mechanisms how tumor cells gain entry into the lymphatic system, and it has been generally thought that lymphatic endothelium only plays a passive role during this process and that lymphatic invasion only occurs once stroma-infiltrating tumor cells happen upon preexisting peritumoral lymphatic vessels. In 2001, we discovered the first direct evidence for active tumor lymphangiogenesis, demonstrating that overexpression of the growth factor VEGF-C by breast cancer cells resulted in enhanced lymphangiogenesis and lymph node metastasis [8]. These findings led to a new model of cancer progression (Fig. 1) and suggested tumor lymphangiogenesis as an important new prognostic parameter and therapeutic target [9]; however, its relevance for human cancers had remained unknown. Our recent results reveal that tumor lymphangiogenesis indeed serves as a novel prognostic indicator for lymph node metastasis and overall patient survival in human malignant melanomas [10]. Ongoing studies investigate the correlation between tumor lymphangiogenesis and metastasis in other human cancers including renal cancer, and the therapeutic potential of inhibition of lymphangiogenesis. We are particularly interested in identifying new therapeutic targets to block metastatic cancer spread and to inhibit the growth of existing lymph node metastases. To this end, we have developed a co-culture system for metastastic and nonmetastatic cancer cells with lymphatic or blood vascular endothelial cells. This model allows us to screen - using genomics and proteomics approaches - for the distinct effects of metastatic cancer cells on lymphatic endothelium, and for the effects of lymphatic endothelium-derived factors on the activation of metastatic cancer cells, potentially leading to the identification of novel targets for advanced cancer therapy.

\section{Transcriptional Profiling for Target Identification}

In contrast to the extensive molecular and functional characterization of tumor 
angiogenesis, little is known about the mechanisms by which tumor cells gain entry into the lymphatic system, mainly due to the lack of reliable markers for the detection of lymphatic endothelium and to the absence of identified mediators of lymphatic vessel formation [11]. To identify new molecular markers for lymphatic (and blood vascular) endothelium, we have developed a new method for the selective isolation and culture of human blood vascular and lymphatic endothelial cells and found that these cells maintain their lineage-specific differentiation even after multiple passages in vitro. Using transcriptional profiling studies combined with quantitative realtime RT-PCR, mouse embryonic stem cell differentiation assays and genetic studies, we have identified more than 300 lymphatic-specific and blood vessel-specific genes and have characterized the specific expression of several of these genes. These studies have led to a more comprehensive understanding of the molecular control of lymphatic and blood vessel growth (Fig. 4). We found that one of the most highly lymphatic-specific genes, podoplanin, plays an important role in lymphatic function [12]. We are investigating whether these genes are also involved in the pathogenesis of human lymphedema and of inflammatory diseases, and whether some of these gene products might serve as novel targets to inhibit tumor angiogenesis, lymphangiogenesis and metastasis. Interestingly, we recently found, for the first time, that cancer viruses can re-program the vascular transcriptome, potentially identifying new molecular targets for the treatment of AIDS-associated malignancies [13].

\section{New Approaches to Treat Inflamma- tory Diseases}

Psoriasis is a chronic inflammatory skin disease that affects approximately $2 \%$ of the population. In 1994, we identified VEGF-A as the major factor responsible for the vascular pathology of psoriasis [14]. We have then developed a transgenic mouse model for targeted overexpression of VEGF-A in the skin, and have discovered that VEGF-A overexpressing transgenic mice are unable to downregulate experimentally induced inflammation and that they develop a chronic inflammatory disease that almost completely resembles human psoriasis [15], therefore representing a new animal model for this disease. Importantly, we found that blockade of VEGF-A signaling potently inhibited inflammation. These findings have stimulated developments by the pharmaceutical industry to develop anti-VEGF therapies for human psoriasis and other inflammatory diseases, and the VEGF transgenic mouse model has

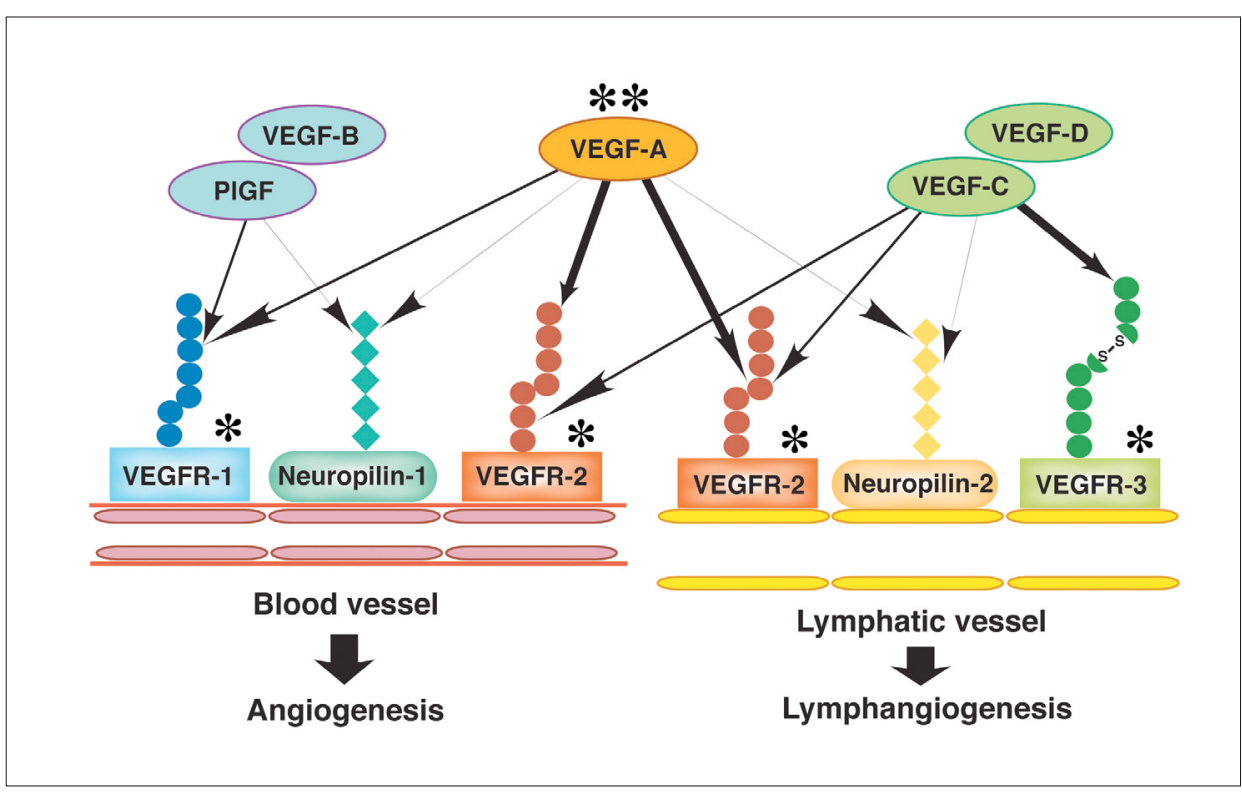

Fig. 4. Schematic representation of specific growth factors and of their receptor expression by lymphatic and blood vascular endothelium. Asterisks identify targets of current FDA-approved (two asterisks) or of experimental anti-cancer therapy.

\section{The VEGF Gene}

\section{Chromosome 6p 21}
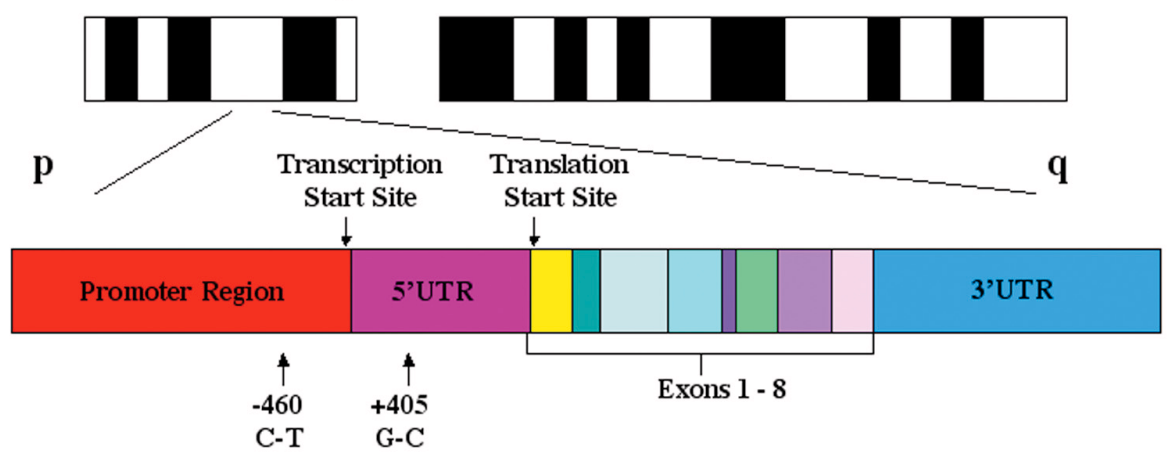

Fig. 5. Schematic representation of the VEGF-A gene and of single nucleotide polymorphisms with relevance to angiogenesis and chronic skin inflammation

been licensed to a number of drug companies for screening of anti-inflammatory compounds. Our ongoing research aims to take advantage of this disease model to further elucidate the molecular mechanisms of chronic inflammation.

\section{Genetic Polymorphisms, Disease Susceptibility, and Pharmacoge- nomics}

Recent evidence indicates that genetic polymorphisms of distinct genes within the general population are responsible for the susceptibility to many of the major chronic diseases, and that they might also be re- sponsible for an individual's response towards drug therapy. Recent studies indicate that distinct single nucleotide polymorphisms (SNPs) of the VEGF gene are associated with reduced or increased levels of circulating VEGF in normal individuals. In particular, the $\mathrm{G}$ to $\mathrm{C}$ polymorphism at position +405 within the leader sequence of the VEGF gene enhances the activity of internal ribosomal entry site $\mathrm{B}$ (IRES-B) and promotes the translation of large VEGF (LVEGF) isoforms at an alternative CUG1 codon (Fig. 5). These L-VEGF isoforms are 205 amino acid residues longer than the AUG initiated VEGF isoforms and appear to increase the intracellular VEGF protein pool. The $+405 \mathrm{C} / \mathrm{C}$ genotype of the VEGF 
gene, and the $+405 \mathrm{C}$ allele, are found significantly more often in patients with severe psoriasis, as compared with healthy control subjects. These findings indicate that distinct genetic polymorphisms might contribute to enhanced VEGF production and to individually increased psoriasis susceptibility. Therefore, the individual 'angiogenetic constitution' appears to determine disease susceptibility [16]. Taken together, therapeutic blockade of the VEGFA/VEGF-A receptor system might represent a novel, pharmacogenomic approach for the future treatment of psoriasis, and we aim to identify individuals that might be particularly sensitive to anti-VEGF-A therapies.

\section{Perspectives}

Angiogenesis and lymphangiogenesis are currently receiving tremendous scientific and clinical interest as novel targets for the treatment of cancer and of inflammatory diseases. The identification and characterization of novel mediators of lymphatic and blood vessel growth and function will likely lead to the development of new therapies for a number of human diseases. Comprehensive research strategies include target identification by transcriptional profiling, validation by genetic models, and establishment of highthroughput screening assays. The identification of genetic polymorphisms that predispose to the development of clinical disease and to the positive response (or adverse effects) to specific drug therapies will likely lead to the future development of individualized, molecular pharmacogenomic therapies for cancer and many other human diseases, avoiding potentially life-threatening adverse effects and providing enhanced efficiency.

\section{Acknowledgments}

The research of the Detmar group is supported by the ETH Zürich, the National Institutes of Health/National Cancer Institute (USA), the American Cancer Society, the Deutsche Forschungsgemeinschaft and the Deutsche Krebshilfe.

August 8, 2004

[1] M. Detmar, J. Investig. Dermatol. Symp. Proc. 2000, 5, 20.

[2] M. Streit, L. Riccardi, P. Velasco, L.F. Brown, T. Hawighorst, P. Bornstein, M. Detmar, Proc. Natl. Acad. Sci. USA 1999, 96, 14888.

[3] T. Hawighorst, P. Velasco, M. Streit, T.R. Kyriakides, L.F. Brown, P. Bornstein, M. Detmar, EMBO J. 2001, 20, 2631.

[4] Y.H. Noh, K. Matsuda, Y.K. Hong, R. Kunstfeld, L. Riccardi, M. Koch, H. Oura, S.S. Dadras, M. Streit, M. Detmar, J. Invest. Dermatol. 2003, 121, 1535.

[5] M. Streit, A.E. Stephen, T. Hawighorst, K. Matsuda, B. Lange-Asschenfeldt, L.F. Brown, J.P. Vacanti, M. Detmar, Cancer Res. 2002, 62, 2004.

[6] T. Hawighorst, H. Oura, M. Streit, L. Janes, L. Nguyen, L.F. Brown, G. Oliver, D.G.
Jackson, M. Detmar, Oncogene 2002, 21, 7945.

[7] K. Yano, H. Oura, M. Detmar, J. Invest. Dermatol. 2002, 118, 800.

[8] M. Skobe, T. Hawighorst, D.G. Jackson, R. Prevo, L. Janes, P. Velasco, L. Riccardi, K. Alitalo, K. Claffey, M. Detmar, Nat. Med. 2001, 7, 192.

[9] M. Detmar, S. Hirakawa, J. Exp. Med. 2002, 6, 713 .

[10] S.S. Dadras, T. Paul, J. Bertoncini, L.F. Brown, A. Muzikansky, D.G. Jackson, U. Ellwanger, C. Garbe, M.C. Mihm, M. Detmar, Am. J. Pathol. 2003, 162, 1951.

[11] G. Oliver, M. Detmar, Genes Dev. 2002, $16,773$.

[12] V. Schacht, M.I. Ramirez, Y.K. Hong, S. Hirakawa, D. Feng, N. Harvey, M. Williams, A.M. Dvorak, H.F. Dvorak, G. Oliver, M. Detmar, EMBO J. 2003, 22, 3546.

[13] Y.K. Hong, K. Foreman, J.W. Shin, S. Hirakawa, C.L. Curry, D.R. Sage, T. Libermann, B.J. Dezube, J.D. Fingeroth, M. Detmar, Nat. Genet. 2004, 36, 683.

[14] M. Detmar, L.F. Brown, K.P. Claffey, K.T. Yeo, O. Kocher, R.W. Jackman, B. Berse, H.F. Dvorak, J. Exp. Med. 1994, 180, 1141.

[15] R. Kunstfeld, S. Hirakawa, Y.K. Hong, V. Schacht, B. Lange-Asschenfeldt, P. Velasco, C. Lin, E. Fiebieger, X. Wei, Y. Wu, D. Hicklin, P. Bohlen, M. Detmar, Blood 2004, 104, 1048.

[16] M. Detmar, J. Invest. Dermatol. 2004, 122, XIV. 